PROCEEDINGS OF THE

AMERICAN MATHEMATICAL SOCIETY

Volume 139, Number 9, September 2011, Pages 3383-3392

S 0002-9939(2011)10958-4

Article electronically published on March 2, 2011

\title{
MIXING CATEGORIES
}

\author{
JOHN OPREA AND JEFF STROM
}

(Communicated by Alexander N. Dranishnikov)

\begin{abstract}
We show that $\operatorname{cat}(X) \leq \operatorname{cat}_{1}(X)+\operatorname{cat}^{1}(X)$, where $\operatorname{cat}_{1}(X)$ is Fox's 1-dimensional category and $\operatorname{cat}^{1}(X)$ is the $\mathcal{A}$-category of Clapp and Puppe with $\mathcal{A}=\{1$-dimensional spaces $\}$.
\end{abstract}

\section{INTRODUCTION}

The Lusternik-Schnirelmann category of a space $X$, denoted cat $(X)$, is the smallest integer $k$ so that $X$ can be covered by open sets $U_{0}, U_{1}, \ldots, U_{k}$, each of which is contractible to a point in $X$. Such a covering is called a categorical covering. LS category is an important numerical invariant in algebraic topology, critical point theory and symplectic geometry (see, for instance, $\mathrm{CLOT}, \mathrm{CP}, \mathrm{RO}]$ ). Since it is notoriously difficult to compute, many approximating invariants have been introduced in order to estimate category from below and above. In this paper, we will mix together two of these approximating invariants to provide the following new upper bound for Lusternik-Schnirelmann category.

Theorem. Writing $\operatorname{cat}_{1}(X)$ for Fox's 1-dimensional category and $\operatorname{cat}^{1}(X)$ for the $\mathcal{A}$-category of Clapp and Puppe with $\mathcal{A}=\{1$-dimensional spaces $\}$,

$$
\operatorname{cat}(X) \leq \operatorname{cat}_{1}(X)+\operatorname{cat}^{1}(X)
$$

for any normal space $X$.

This has, as a direct consequence, a result recently proved in Dr2] (and re-proved in $\mathrm{OS}$ ).

Corollary. If $\pi=\pi_{1}(X)$ has finite cohomological dimension, then

$$
\operatorname{cat}(X) \leq \operatorname{dim}(B \pi)+\left\lceil\frac{\operatorname{dim}(X)-1}{2}\right\rceil .
$$

The theorem above thus provides a more general underlying reason for the truth of the corollary than what has been presented before. Nevertheless, it is the classical open set methods introduced in Dr1, Dr2 that demonstrate the theorem. It is worth mentioning that we have repackaged Dranishnikov's rather clever and intricate construction into an easily understood lemma, which may prove useful outside the present paper.

Received by the editors August 22, 2010.

2010 Mathematics Subject Classification. Primary 55M30; Secondary 55P99.

Key words and phrases. Lusternik-Schnirelmann category.

(C)2011 American Mathematical Society Reverts to public domain 28 years from publication 
Lemma 4.3. Let $X$ be a normal space with two open covers

$$
\mathcal{U}=\left\{U_{0}, U_{1}, \ldots, U_{k}\right\} \quad \text { and } \quad \mathcal{V}=\left\{V_{0}, V_{1}, \ldots, V_{m}\right\}
$$

such that each set of $\mathcal{U}$ satisfies Property $(A)$, and each set of $\mathcal{V}$ satisfies Property $(B)$. Assume that Properties $(A)$ and $(B)$ are preserved under taking open subsets and disjoint unions. Then $X$ has an open cover

$$
\mathcal{W}=\left\{W_{0}, W_{1}, \ldots, W_{k+m}\right\}
$$

by open sets satisfying both Property (A) and Property (B).

The theorem above begs the question whether $\operatorname{cat}(X) \leq \operatorname{cat}_{m}(X)+\operatorname{cat}^{m}(X)$, where $m>1$ (and $\mathrm{cat}_{m}$, cat ${ }^{m}$ are defined below) or indeed, $\operatorname{cat}(X) \leq \operatorname{cat}_{m}(X)+$ cat $^{n}(X)$, for any $m, n>0$. In the final section we begin the general discussion of these questions, leading to a qualitative description of the geography of mixed categories.

Throughout the paper, we consider spaces that are paracompact normal ANR's (see [CLOT, Appendix 1]).

\section{Fox's $m$-DIMENSIONAL CATEGORY}

In $[\mathrm{Fo}, \mathrm{R}$. Fox introduced the notion of $m$-dimensional category as an approximating invariant for LS category. We say that $\operatorname{cat}_{m}(X)=k$ if $k$ is the least integer so that there exists an open cover $\left\{U_{0}, \ldots, U_{k}\right\}$ of $X$ such that, for each $U_{j}$, every composition $P \rightarrow U_{j} \hookrightarrow X$ with $\operatorname{dim}(P) \leq m$ is nullhomotopic. We say that any such open set $U$ is $m$-categorical. Immediately, we see that $\operatorname{cat}_{m}(X) \leq \operatorname{cat}(X)$ for all $m \geq 0$.

We write $X \rightarrow X[m]$ for the $m^{\text {th }}$ Postnikov section of $X$ and $X\langle m\rangle \rightarrow X$ for its homotopy fiber, known as the $m$-connected cover of $X$ (in particular, $X\langle 1\rangle \rightarrow X$ is, up to homotopy equivalence, the universal cover of $X$ ). Svarc Sv identified $\operatorname{cat}_{m}(X)$ with an invariant called the genus of the $m$-connected cover fibration $X\langle m\rangle \rightarrow X$. In modern parlance, the genus of a fibration $F \rightarrow E \stackrel{p}{\rightarrow} B$ is called the sectional category; it is the least integer $k$ for which there is an open cover $B=U_{0} \cup U_{1} \cup \cdots \cup U_{k}$ such that there is a partial section of $p$ over each $U_{j}$. Thus we have the following modern formulation of Svarc's result (also see CP, Proposition 4.4]).

Proposition 2.1 ( $\underline{\mathrm{Sv}}$, Proposition 44]). $\operatorname{cat}_{m}(X)=\operatorname{secat}(X\langle m\rangle \rightarrow X)$.

Therefore $m$-categorical open sets have the property that their inclusion maps lift through the $m$-connected cover of the ambient space. The following consequence is implicit in the more complicated results of [EG, LM].

Corollary 2.2. If $\pi_{1}(X)=\pi$ and $B \pi=K(\pi, 1)$, then

$$
\operatorname{cat}_{1}(X) \leq \operatorname{cat}(B \pi)=\operatorname{dim}(B \pi) .
$$

Moreover, if $X=K(\pi, 1)$, then $\operatorname{cat}_{1}(X)=\operatorname{cat}(B \pi)=\operatorname{dim}(B \pi)$.

Proof. If $\operatorname{dim}(B \pi)=\infty$, then there is nothing to prove, so assume $\operatorname{dim}(B \pi)$ is finite. By [EG], we know $\operatorname{dim}(B \pi)=\operatorname{cat}(B \pi)$, so suppose $\operatorname{cat}(B \pi)=k$ with categorical open cover $\left\{U_{0}, \ldots, U_{k}\right\}$. Consider the open cover of $X$ given by $\left\{q^{-1}\left(U_{j}\right) \mid j=\right.$ $0, \ldots, k\}$, where $q: X \rightarrow B \pi$ is the classifying map for the universal cover. Now, $q\left(q^{-1}\left(U_{j}\right)\right) \subseteq U_{j}$ is categorical since $U_{j}$ contracts to a point in $B \pi$. Therefore, up to homotopy, the inclusion $q^{-1}\left(U_{j}\right) \hookrightarrow X$ lifts through the fibre of $q$, which is, up 
to homotopy, the universal cover $\widetilde{X}$. Then, by Proposition 2.1 with $m=1$, we see that $\left\{q^{-1}\left(U_{j}\right)\right\}$ is a 1-categorical cover of $X$. Hence $\operatorname{cat}_{1}(X) \leq k$.

Now assume $X=K(\pi, 1)=B \pi$, and note that $\widetilde{X}=X\langle 1\rangle$ is contractible. If $\left\{U_{0}, \ldots, U_{k}\right\}$ is a cat ${ }_{1}$-cover of $X$, then each inclusion $U_{j} \hookrightarrow X$ factors through the universal cover $\tilde{X} \simeq *$ up to homotopy, so $\left\{U_{0}, \ldots, U_{k}\right\}$ is actually a categorical cover. Thus $\operatorname{dim}(B \pi)=\operatorname{cat}(X) \leq \operatorname{cat}_{1}(X)$ and combining with the general inequality $\operatorname{cat}_{1}(X) \leq \operatorname{dim}(B \pi)$ gives an equality.

The same argument shows that if $\pi_{n}(X)=0$ for $n>m$, then $\operatorname{cat}_{m}(X)=\operatorname{cat}(X)$. The estimate $\operatorname{cat}_{1}(X) \leq \operatorname{dim}(B \pi)$ has the following immediate consequence.

Corollary 2.3. If $\pi_{1}(X)$ is a non-trivial free group, then cat $_{1}(X)=1$.

Proof. The only thing to check is that we cannot have $\operatorname{cat}_{1}(X)=0$, but this follows because $\operatorname{cat}_{1}(X)=0$ would imply that the universal covering $\widetilde{X} \rightarrow X$ has a section and this can only happen if $\pi_{1}(X)$ is trivial.

In fact, it is true that $\pi_{1}(X)$ is free if and only if $\operatorname{cat}_{1}(X)=1$. This follows from a characterization of cat ${ }_{1}$ established in [EG] (also see [LM]). Namely, $\operatorname{cat}_{1}(X) \leq n$ if and only if there is an $n$-dimensional complex $L$ and a map $X \rightarrow L$ which induces an isomorphism on fundamental groups. If $\operatorname{cat}_{1}(X)=1$, this implies $\pi_{1}(X) \cong \pi_{1}(L)$ with $\operatorname{dim}(L)=1$, and the fundamental group of any 1-dimensional complex is free.

\section{The Clapp-Puppe $\mathcal{A}$-Category}

Now let us look at a "dual" to $m$-dimensional category cat $_{m}$. Following Clapp and Puppe [CP] 11 we define the $\mathcal{A}$-category of $X$. An open cover $\mathcal{U}=\left\{U_{0}, U_{1}\right.$, $\left.\ldots, U_{k}\right\}$ of $X$ is a categorical $\mathcal{A}$-cover if each inclusion $U_{j} \hookrightarrow X$ factors, up to homotopy, through a space $A \in \mathcal{A}$. Then $\operatorname{cat}_{\mathcal{A}}(X)$ is the least integer $k$ so that $\mathcal{U}$ is a categorical $\mathcal{A}$-cover of $X$.

Clapp and Puppe single out the case $\mathcal{A}_{r}=\{r$-dimensional spaces $\}$ as worthy of special attention and they suggest the notation cat $^{r}$ for this special notion of category. For path-connected spaces, cat $^{0}$ is the ordinary LS category. Also, if $s \leq r$, then $\mathcal{A}_{s} \subseteq \mathcal{A}_{r}$ and so $\operatorname{cat}^{r}(X) \leq \operatorname{cat}^{s}(X)$. In particular, $\operatorname{cat}^{r}(X) \leq \operatorname{cat}(X)$ for any space $X$ and $\operatorname{cat}^{r}(X)=\operatorname{cat}(X)$ if $X$ is $r$-connected (since the $r$-skeleton of $X$ can be taken to be a point then). Estimates for $\operatorname{cat}^{r}(X)$ are established in [CP,, $\mathrm{OS}$.

Proposition 3.1 ([CP,,$\overline{\mathrm{OS}}]$ ). Let $X$ be a space with $r$-skeleton $X_{r}$. Suppose that $h^{*}$ is a multiplicative cohomology theory. If $K=\operatorname{ker}\left(h^{*}(X) \rightarrow h^{*}\left(X_{r}\right)\right)$, then

$$
\operatorname{nil}(K) \leq \operatorname{cat}^{r}(X) \leq\left\lfloor\frac{\operatorname{dim}(X)}{r+1}\right\rfloor
$$

where $\operatorname{nil}(K)$ is the length of the longest non-trivial product of degree non-zero elements in $K$.

A closed manifold $X^{2 n}$ is said to be cohomologically symplectic (or c-symplectic) if there is a class $\omega \in H^{2}(X ; \mathbb{R})$ such that $\omega^{n} \in H^{2 n}(X ; \mathbb{R}) \cong \mathbb{R}$ is non-trivial. Every

\footnotetext{
${ }^{1}$ Note that we use reduced category, where $\operatorname{cat}_{\mathcal{A}}(*)=0$, while Clapp and Puppe use unreduced category, where $\operatorname{cat}_{\mathcal{A}}(*)=1$.
} 
symplectic manifold is, of course, c-symplectic. Note that the standard cuplength and dimension inequalities for LS category imply that

$$
n \leq \operatorname{cat}(X) \leq 2 n .
$$

We now compute the 1-category of c-symplectic manifolds.

Proposition 3.2. A c-symplectic manifold $\left(X^{2 n}, \omega\right)$ has $\operatorname{cat}^{1}(X)=n=\frac{1}{2} \operatorname{dim}(X)$.

Proof. By Proposition 3.1, we have

$$
n \leq \operatorname{nil}(K) \leq \operatorname{cat}^{1}(X) \leq\left\lfloor\frac{\operatorname{dim}(X)}{1+1}\right\rfloor=\left\lfloor\frac{2 n}{2}\right\rfloor=n,
$$

since $\omega \in K=\operatorname{ker}\left(H^{*}(X ; \mathbb{R}) \rightarrow H^{*}\left(X_{1} ; \mathbb{R}\right)\right)$.

\section{Generalities on OPEN COVERS}

The main results about open covers that we shall use are described (and proved) in [Dr1, Dr2, but other relevant papers include $\mathrm{O}, \mathrm{Ha}, \mathrm{Cu}$ as well as [CLOT, Exercise 1.12].

An open cover $\mathcal{W}=\left\{W_{0}, \ldots, W_{m+k}\right\}$ of a space $X$ is an $(m+1)$-cover if every subcollection $\left\{W_{j_{0}}, W_{j_{1}}, \ldots, W_{j_{m}}\right\}$ of $m+1$ sets from $\mathcal{U}$ also covers $X$. The following simple, but slippery, observation (see [O] for instance) is often given without proof, but it is the basis for many arguments in this approach.

Lemma 4.1. A cover $\mathcal{W}=\left\{W_{0}, W_{1}, \ldots, W_{k+m}\right\}$ is an $(m+1)$-cover of $X$ if and only if each $x \in X$ is contained in at least $k+1$ sets of $\mathcal{W}$.

Proof. If $\mathcal{W}$ is an $(m+1)$-cover and $x \in X$ is only in $k$ sets in $\mathcal{W}$, then $k+m+1-k=$ $m+1$ sets of the cover do not contain $x$. These $m+1$ sets do not cover $X$, contradicting the supposition on $\mathcal{W}$.

Suppose each $x \in X$ is contained in at least $k+1$ sets from $\mathcal{W}$ and choose a subcollection $\mathcal{V}$ of $m+1$ sets from $\mathcal{W}$. There are only $k+m+1-(m+1)=k$ sets not in $\mathcal{V}$, so $x$ must belong to at least one set in $\mathcal{V}$. Thus $\mathcal{V}$ covers $X$, and $\mathcal{W}$ is an $(m+1)$-cover.

An open cover can be lengthened to a $(k+1)$-cover, while retaining certain essential properties of the sets in the cover.

Theorem $4.2([\mathrm{Cu}, \overline{D r} 1])$. Let $\mathcal{U}=\left\{U_{0}, \ldots, U_{k}\right\}$ be an open cover of a normal space $X$. Then, for any $m=k, k+1, \ldots, \infty$, there is an open $(k+1)$-cover of $X$, $\left\{U_{0}, \ldots, U_{m}\right\}$, extending $\mathcal{U}$ such that for $n>k, U_{n}$ is a disjoint union of open sets that are subsets of the $U_{j}, 0 \leq j \leq k$.

In Theorem 4.2, because the $U_{n}$ for $n>k$ are disjoint unions of subsets of the original covering sets, the $U_{n}$ also possess any properties of the original cover that are inherited by disjoint unions and open subsets. In particular, if the cover $\mathcal{U}$ is categorical (or $m$-categorical, or $\mathcal{A}$-categorical), then the extended cover is also categorical (or $m$-categorical, or $\mathcal{A}$-categorical).

Lemma 4.3. Let $X$ be a normal space with two open covers

$$
\mathcal{U}=\left\{U_{0}, U_{1}, \ldots, U_{k}\right\} \quad \text { and } \quad \mathcal{V}=\left\{V_{0}, V_{1}, \ldots, V_{m}\right\}
$$


such that each set of $\mathcal{U}$ satisfies Property $(A)$ and each set of $\mathcal{V}$ satisfies Property (B). Assume that Properties (A) and (B) are inherited by open subsets and disjoint unions. Then $X$ has an open cover

$$
\mathcal{W}=\left\{W_{0}, W_{1}, \ldots, W_{k+m}\right\}
$$

by open sets satisfying both Property (A) and Property (B).

Proof. Using Theorem 4.2, extend $\mathcal{U}$ to a $(k+1)$-cover $\widetilde{\mathcal{U}}=\left\{U_{0}, \ldots, U_{k+m}\right\}$ and extend $\mathcal{V}$ to an $(m+1)$-cover $\widetilde{\mathcal{V}}=\left\{V_{0}, \ldots, V_{k+m}\right\}$. Since each set in $\widetilde{\mathcal{U}}$ is a disjoint union of open subsets of sets in $\mathcal{U}$, the cover $\widetilde{\mathcal{U}}$ consists of sets satisfying Property (A); likewise, each set in $\widetilde{\mathcal{V}}$ satisfies Property (B). Since Properties (A) and (B) are inherited by open subsets and disjoint unions, we see that each set $U_{i} \cap V_{j}$ satisfies both properties.

Therefore, the lemma will be proved if we can show that the collection

$$
\mathcal{W}=\left\{U_{0} \cap V_{0}, U_{1} \cap V_{1}, \ldots, U_{k+m} \cap V_{k+m}\right\}
$$

is an open cover of $X$. First, observe that since $\widetilde{V}$ is an $(m+1)$-cover, each point $x \in X$ lies in at least $k+1$ sets of $\widetilde{\mathcal{V}}$; we may suppose, without loss of generality, that $x \in V_{0} \cap \cdots \cap V_{k}$. Next, since $\widetilde{\mathcal{U}}$ is a $(k+1)$-cover, the subcollection $\left\{U_{0}, \ldots, U_{k}\right\}$ covers $X$, and so $x \in U_{i}$ for some $0 \leq i \leq k$. Thus $x \in U_{i} \cap V_{i}$ for at least one value of $i$ and $\mathcal{W}$ covers $X$.

\section{Mixing CATEGories}

We now wish to apply our results about open covers to obtain an estimate for LS category.

Theorem 5.1. If $X$ is a normal space, then

$$
\operatorname{cat}(X) \leq \operatorname{cat}_{1}(X)+\operatorname{cat}^{1}(X) .
$$

Proof. Suppose $\operatorname{cat}_{1}(X)=k$ and $\operatorname{cat}^{1}(X)=m$. Let $\mathcal{U}=\left\{U_{0}, \ldots, U_{k}\right\}$ be a cat ${ }^{-}$ open cover of $X$ and let $\mathcal{V}=\left\{V_{0}, \ldots, V_{m}\right\}$ be a cat ${ }^{1}$-open cover of $X$. Thus, each inclusion $U_{j} \hookrightarrow X$ lifts through the universal cover $\tilde{X}$, while each inclusion $V_{j} \hookrightarrow X$ may be homotopically factored through a 1-dimensional space $Z$ and hence through the 1-skeleton of $X$, denoted $X_{1}$ (which we can take to be a wedge of circles). By Lemma 4.3, we have an open cover $\mathcal{W}=\left\{W_{0}, \ldots, W_{k+m}\right\}$ such that each inclusion $W_{j} \hookrightarrow X$ satisfies both properties.

We claim that any open subset of $X$ that is both a cat ${ }^{1}$-set and a cat ${ }_{1}$-set is actually categorical. Consider the inclusion $i_{j}: W_{j} \hookrightarrow X$ and note that, since $i_{j}$ factors through $\tilde{X}$, we have $i_{j *}\left(\pi_{1}\left(W_{j}\right)\right)=\{1\}$. (If $W_{j}$ is not connected, we do this component by component.) But then we consider the factorization of $i_{j}$ through $X_{1}, W_{j} \stackrel{\sigma}{\rightarrow} X_{1} \hookrightarrow X$ and infer that $\operatorname{Im}\left(\sigma_{*}\right) \subseteq K=\operatorname{Ker}\left(\pi_{1}\left(X_{1}\right) \rightarrow \pi_{1}(X)\right)$. Now take the covering space $X_{K} \rightarrow X_{1}$ of $X_{1}$ corresponding to $K$ and note that, since $X_{1} \simeq \vee S^{1}$, the same is true for $X_{K}$ (or any covering space). But then we see that the composition $X_{K} \rightarrow X_{1} \hookrightarrow X$ is nullhomotopic because it is zero (by definition of $K$ ) on fundamental groups and $X_{K}$ is a wedge of $S^{1}$ 's. Also, because $\operatorname{Im}\left(\sigma_{*}\right) \subseteq K$, 
we obtain a lift $\phi$ in the following homotopy commutative diagram:

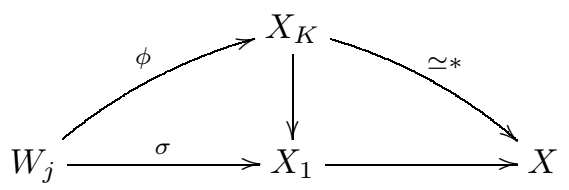

But the bottom row is $i_{j}$, so that $i_{j} \simeq *$. Therefore, each inclusion $i_{j}: W_{j} \rightarrow X$ is nullhomotopic, and we see that the open cover $\mathcal{W}$ is categorical.

The general result, Theorem [5.1, provides a unification for two estimates of LS category, one classical and one recent.

Corollary 5.2 (I. Berstein; see $[\underline{\mathrm{Sv}}$ ).

$$
\operatorname{cat}(X) \leq \operatorname{cat}_{1}(X)+\left\lfloor\frac{\operatorname{dim}(X)}{2}\right\rfloor .
$$

Proof. Apply the estimate of Proposition 3.1 with $r=1$.

In fact, Berstein's estimate holds in the generality

$$
\operatorname{cat}(X) \leq \operatorname{cat}_{m}(X)+\left\lfloor\frac{\operatorname{dim}(X)}{m+1}\right\rfloor
$$

which begs the question of whether it is true that $\operatorname{cat}(X) \leq \operatorname{cat}_{m}(X)+\operatorname{cat}^{m}(X)$. We shall discuss this more in $\sqrt{6}$.

Corollary 5.3 (see Dr2,

$$
\operatorname{cat}(X) \leq \operatorname{dim}(B \pi)+\operatorname{cat}^{1}(X) \leq \operatorname{dim}(B \pi)+\left\lfloor\frac{\operatorname{dim}(X)}{2}\right\rfloor,
$$

where $B \pi=K(\pi, 1)$ and $\pi=\pi_{1}(X)$ 2

Proof. Apply Corollary 2.2 to $\operatorname{cat}_{1}(X)$ in Theorem 5.1 .

Note that Theorem 5.1 has the huge advantage over Corollary 5.3 that $\operatorname{cat}_{1}(X)+$ cat $^{1}(X)<\infty$ for typical $X$ (e.g. if $\operatorname{dim}(X)<\infty$ ) while $\operatorname{dim}(B \pi)=\infty$ for most groups (e.g. any group with torsion).

By Corollary 2.3 and Proposition 3.2, we obtain the following.

Corollary 5.4. If $\left(X^{2 n}, \omega\right)$ is c-symplectic and $\pi_{1}(X)$ is a free group, then $n \leq$ $\operatorname{cat}(X) \leq n+1$.

\section{The GEOGRAPHy OF CATEGORIES}

It appears that Theorem 5.1 is mixing together two very different "projections" of category to produce a single picture (or at least an approximate picture) of it. Can other pairs of invariants be combined to bound LS category? The argument proving Theorem 5.1 uses fundamental properties of 1-skeleta and their coverings and does not work for $\mathrm{cat}_{m}$ and $\mathrm{cat}^{n}$. Indeed, the following example indicates that such an argument cannot work.

\footnotetext{
${ }^{2} \operatorname{In}\lceil\overline{D r 2}$, the bound $\lceil(\operatorname{dim}(X)-1) / 2\rceil$ is used, but note that for an integer $r>1$ and any integer $n,\lfloor n / r\rfloor \leq\lceil(n-1) / r\rceil$ with equality holding when $r=2$.
} 
Example 6.1. Consider the following homotopy commutative diagram:

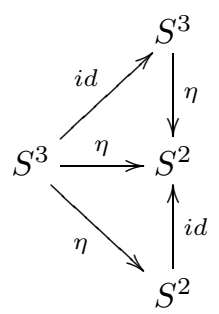

We can fatten the middle row to be the inclusion of an open set into a space of the homotopy type of $S^{2}$. But then we see that the open set both lifts to the 2-connected cover of $S^{2}$ (i.e. $S^{3}$ ) and compresses into the 2-skeleton of $S^{2}$ (i.e. $S^{2}$ itself). But the middle row is not nullhomotopic since it is the Hopf map $\eta$.

This example indicates that we cannot generalize the proof of Theorem 5.1 to obtain the more general result $\operatorname{cat}(X) \leq \operatorname{cat}_{m}(X)+\operatorname{cat}^{m}(X)$. Instead, we take a more global point of view and consider the set

$$
\mathcal{C}(X)=\left\{(m, n) \mid \operatorname{cat}(X) \leq \operatorname{cat}_{m}(X)+\operatorname{cat}^{n}(X)\right\} .
$$

The assignment $X \mapsto \mathcal{C}(X)$ is a strange new homotopy invariant that gives us information about a space by displaying the interplay between its Fox and ClappPuppe categories. Of course, our hope is that (at least some features of) $\mathcal{C}(X)$ may be understood without explicit calculation of the various $\mathrm{cat}_{m}$ and $\mathrm{cat}^{n}$, but for the present paper, we are content to establish some elementary properties.

Proposition 6.2. Let $X$ be a normal d-dimensional and c-connected space with $c \geq 0$. Then we have the following:

(1) $(1,1) \in \mathcal{C}(X)$.

(2) $(m, c) \in \mathcal{C}(X)$ for all $m$.

(3) $(0, n) \in \mathcal{C}(X)$ if and only if $\operatorname{cat}(X)=\operatorname{cat}^{n}(X)$.

(4) Let $n \geq d$. Then $(m, n) \in \mathcal{C}(X)$ if and only if $\operatorname{cat}(X)=\operatorname{cat}_{m}(X)$. In particular, $(m, n) \in \mathcal{C}(X)$ if $m \geq d$.

(5) If $(m, n) \in \mathcal{C}(X)$, then $(m+s, n-t) \in \mathcal{C}(X)$ for all $s, t \geq 0$.

Proof. Part (1) is a restatement of Theorem 5.1 Part (2) follows from the observation that $\operatorname{cat}^{c}(X)=\operatorname{cat}(X)$. Parts (3) and (4) result from the following facts:

- $\operatorname{cat}_{0}(X)=0$;

- cat $^{n}(X), \operatorname{cat}_{m}(X) \leq \operatorname{cat}(X)$ for any $X$;

- $\operatorname{cat}^{n}(X)=0$ if $\operatorname{dim}(X) \leq n$;

- $\operatorname{cat}_{m}(X)=\operatorname{cat}(X)$ for $m \geq \operatorname{dim}(X)$ (see [Fo, 17.2]).

Finally, (5) holds because $\operatorname{cat}_{m}(X)$ increases with increasing $m$ and cat $^{n}(X)$ decreases with increasing $n$.

It follows from Proposition 6.2 that if $X$ is $c$-connected and $d$-dimensional, then the set $\mathcal{C}(X)$ has the form shown in Figure 1, Proposition 6.2(4) guarantees that there can only be finitely many "corner points", and determining the shape of $\mathcal{C}(X)$ ultimately boils down to finding them.

Problem 6.3. For a space $X$, determine the shape of $\mathcal{C}(X)$.

The following example shows that dimension and LS category do not determine the shape of $\mathcal{C}(X)$. 


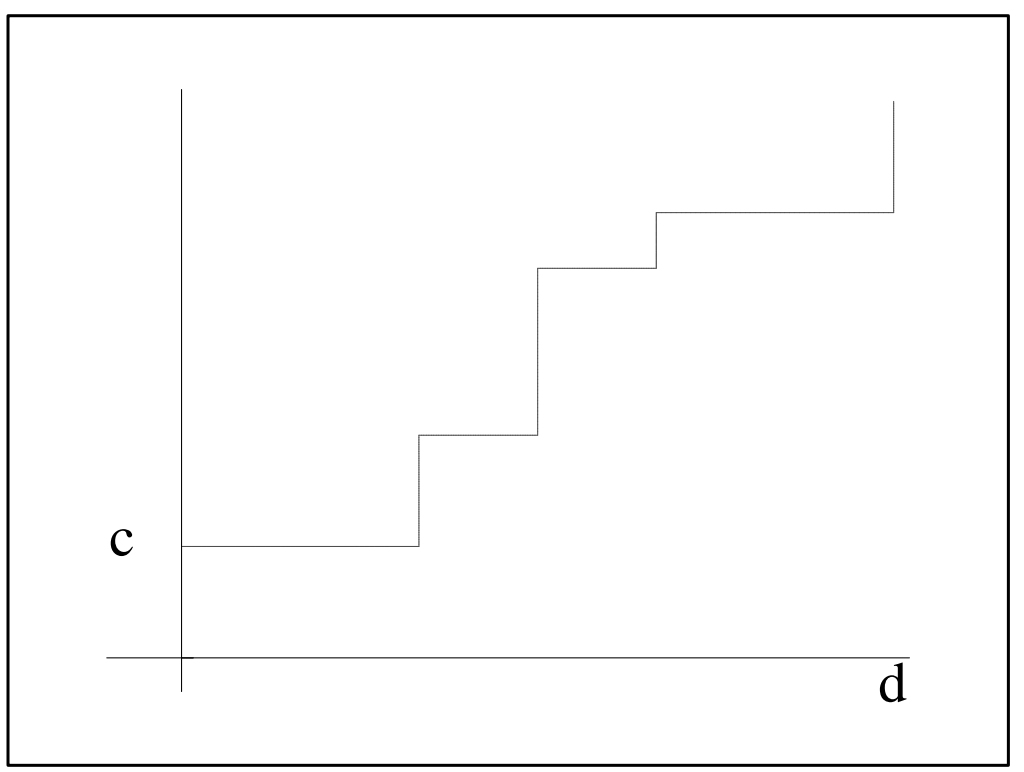

Figure 1. A possible $\mathcal{C}(X)$.

Example 6.4. Let $X=S^{1} \times S^{3}$ and $Y=S^{2} \times S^{2}$. Note that $\operatorname{dim}(X)=4=\operatorname{dim}(Y)$ and $\operatorname{cat}(X)=2=\operatorname{cat}(Y)$ by the standard cuplength and product inequalities for LS category. By Corollary 2.3, we see that $\operatorname{cat}_{1}(X)=1$, while $\operatorname{cat}^{1}(X)=1$ by combining the lower bound of Proposition 3.1 with the explicit covering $\left\{S^{1} \times\right.$ $\left.U_{0}, S^{1} \times U_{1}\right\}$, where $\left\{U_{0}, U_{1}\right\}$ is the standard "hemispherical" categorical covering of $S^{3}$. Clearly, then we see that $(0,1) \notin \mathcal{C}(X)$. The same argument shows that $\operatorname{cat}^{3}(X)=1$, where we now use a covering $\left\{U_{0} \times S^{3}, U_{1} \times S^{3}\right\}$, where $\left\{U_{0}, U_{1}\right\}$ is the standard categorical covering of $S^{1}$. Hence, $(1,3) \in \mathcal{C}(X)$ is a corner point. Since the 2-connected cover of $X$ is the same as its 1-connected cover, we also have $\operatorname{cat}_{2}(X)=1$. However, $(2,4) \notin \mathcal{C}(X)$ since $\operatorname{cat}^{4}(X)=0$. Now, the degree 1 and 3 cohomology classes in $X$ are killed when pulled back to the 3-connected cover and their product in $H^{*}(X)$ is non-trivial. Hence, by [CLOT, Proposition 9.14], $\operatorname{cat}_{3}(X) \geq 2=\operatorname{cat}(X)$. Thus, $\operatorname{cat}_{3}(X)=2$ and $(3,4) \in \mathcal{C}(X)$ is a corner point. The corners are then $\{(1,3),(3,4)\}$.

Now consider $Y$. Because $Y$ is simply connected, we have $\operatorname{cat}^{1}(Y)=\operatorname{cat}(Y)$, and this implies that $(0,1) \in \mathcal{C}(Y)$. Thus, the entire horizontal line $(m, 1) \in \mathcal{C}(Y)$. We also have $\operatorname{cat}^{2}(Y)=1$ since removing a small 4-disk from $Y$ leaves $S^{2} \vee S^{2}$ homotopically. Therefore, $(1,2) \notin \mathcal{C}(Y)$ since $\operatorname{cat}_{1}(Y)=0$ by simple connectivity. Finally, we have $\operatorname{cat}_{2}(Y)=2=\operatorname{cat}(Y)$ (again by [CLOT, Proposition 9.14]), so $(2,4) \in \mathcal{C}(Y)$. Hence, the corners are $\{(0,1),(2,4)\}$, and the shape of $\mathcal{C}(Y)$ is very different from that of $\mathcal{C}(X)$.

Because the finiteness of the set of corner points results from the vertical line guaranteed by Proposition 6.2(4), one might wonder whether this line can sometimes be moved to the left.

Example 6.5. The torus $T^{2}=B(\mathbb{Z} \times \mathbb{Z})$ has a vertical line at $m=1$ since $\operatorname{cat}_{1}\left(T^{2}\right)=\operatorname{cat}\left(T^{2}\right)=2$ by Corollary 2.2 . 
On the other hand, we might ask whether the horizontal bound on $\mathcal{C}(X)$ might be raised or is it entirely governed by the connectivity of $X$ ? Because of Proposition 6.2 $(5)$, there is a horizontal line at height $n$ if and only if $(0, n) \in \mathcal{C}(X)$. Thus we ask: can it be that $(0, n) \in \mathcal{C}(X)$ for $n>\operatorname{conn}(X)$ ? An affirmative answer is given by the following.

Example 6.6. Let $\mathbb{H} \mathrm{P}^{5}$ denote quaternionic projective 5 -space. Because $\mathbb{H} \mathrm{P}^{5}$ is 3 connected, $\operatorname{cat}\left(\mathbb{H} \mathrm{P}^{5}\right)=\operatorname{cat}^{3}\left(\mathbb{H} \mathrm{P}^{5}\right)=5$ (by Proposition 3.1). Now, let $X=\mathbb{H} \mathrm{P}^{5} \vee S^{2}$ and note that $\operatorname{cat}(X)=\mathrm{cat}^{3}(X)=5$ by general properties of categories (see, for instance, [CLOT, Proposition 1.27]). Thus we see that

$$
\operatorname{cat}(X) \leq \operatorname{cat}_{m}(X)+\operatorname{cat}^{3}(X) \quad \text { for all } m
$$

and $3>1=\operatorname{conn}(X)$.

Similar examples, using products, can be constructed using Iwase's counterexamples to the Ganea conjecture and results from [CP2].

\section{REFERENCES}

[CP] M. Clapp and D. Puppe, Invariants of the Lusternik-Schnirelmann type and the topology of critical sets, Trans. Amer. Math. Soc. 298 (1986), no. 2, 603-620. MR860382 (88d:55004)

[CP2] M. Clapp and D. Puppe, The generalized Lusternik-Schnirelmann category of a product space, Trans. Amer. Math. Soc. 321 (1990), no. 2, 525-532. MR1038015 (91a:55012)

[CLOT] O. Cornea, G. Lupton, J. Oprea and D. Tanré, Lusternik-Schnirelmann Category, Amer. Math. Soc. Surveys and Mono. 103 (2003). MR1990857(2004e:55001)

[Cu] M. Cuvilliez, LS-catégorie et k-monomorphisme, Thèse, Université Catholique de Louvain (1998).

[Dr1] A. Dranishnikov, On the Lusternik-Schnirelmann category of spaces with 2-dimensional fundamental group, Proc. Amer. Math. Soc. 137 (2009), no. 4, 1489-1497. MR.2465675 (2009i:55006)

[Dr2] A. Dranishnikov, The Lusternik-Schnirelmann category and the fundamental group, Algebraic \& Geometric Topology 10 (2010), 917-924. MR2629770

[EG] S. Eilenberg and T. Ganea, On the Lusternik-Schnirelmann category of abstract groups, Ann. of Math. (2) 65, no. 3 (1957), 517-518. MR0085510 (19:52d)

[Fo] R. Fox, On the Lusternik-Schnirelmann category, Ann. of Math. (2) 42 (1941), 333-370. MR0004108 (2:320f)

[Ha] K. A. Hardie, On cat ${ }^{i}$ X, J. Lond. Math. Soc. 3 (1971), 91-92. MR0293626 (45:2703)

[LM] E. Laitinen and T. Matumoto, A gap theorem for Lusternik-Schnirelmann $\pi_{1}$-category, Top. and its Apps. 93 (1999), 35-40. MR1684210 (2000c:55006)

[NSS] R. Nendorf, N. Scoville and J. Strom, Categorical sequences, Algebr. Geom. Topol. 6 (2006), 809-838. MR2240916 (2007e:55002)

[O] P. Ostrand, Dimension of metric spaces and Hilbert's problem 13, Bull. Amer. Math. Soc. 71 (1965), 619-622. MR0177391 (31:1654)

[OS] J. Oprea and J. Strom, Lusternik-Schnirelmann category, complements of skeleta and a theorem of Dranishnikov, Algebraic \& Geometric Topology 10 (2010), 1165-1186. MR2653059

[RO] Y. Rudyak and J. Oprea, On the Lusternik-Schnirelmann category of symplectic manifolds and the Arnold conjecture, Math. Z. 230 (1999), no. 4, 673-678. MR1686579 (2000b:53115) 
[Str] J. Strom, Lusternik-Schnirelmann category of spaces with free fundamental group, Algebr. Geom. Topol. 7 (2007), 1805-1808. MR.2366179 (2008k:55007)

[Sv] A. Svarc, The genus of a fiber space, Amer. Math. Soc. Transl. Series 2, vol. 55 (1966), 49-140.

Department of Mathematics, Cleveland State University, Cleveland, Ohio 44115

E-mail address: j.oprea@csuohio.edu

Department of Mathematics, Western Michigan University, Kalamazoo, Michigan 49008-5200

E-mail address: Jeff.Strom@wmich.edu 